\title{
Projection and Aggregation in Maxplus Algebra
}

\author{
G. Cohen ${ }^{1}$, S. Gaubert ${ }^{2}$, and J.-P. Quadrat ${ }^{2}$ \\ 1 ENPC 6-8, avenue Blaise Pascal Cité Descartes, Champs-sur-Marne 77455 \\ Marne-La-Vallée Cedex 2, France Guy.Cohen@enpc.fr \\ 2 INRIA-Rocquencourt, B.P. 105, 78153 Le Chesnay Cedex, France \\ Stephane.Gaubert@inria.Fr and Jean-Pierre.Quadrat@inria.fr
}

Summary. In maxplus algebra, linear projectors on an image of a morphism $B$ parallel to the kernel of another morphism $C$ can be built under transversality conditions of the two morphisms. The existence of a transverse to an image or a kernel of a morphism is obtained under some regularity conditions. We show that those regularity and transversality conditions can be expressed linearly as soon as the space to which $\operatorname{im}(B)$ and $\operatorname{ker}(C)$ belong is free and its order dual is free. The algebraic structure $\overline{\mathbb{R}}_{\max }^{n}$ has these two properties. Projectors are constructed following a previous work. Application to aggregation of linear dynamical systems is discussed.

\section{Introduction}

The work of Peter Kokotovic [9] with François Delebecque and the third author, about aggregation of Markov chains, that we have tried to extend to the maxplus algebra context, has motivated our research on the construction of maxplus linear projectors $[5,6]$. This construction presents some difficulties in this new algebraic context. In this paper, we survey some facts given in these previous papers and clarify some links with module theory.

The practical motivation to study projectors in the context of maxplus algebra is the curse of dimensionality in dynamic programming which is the main restriction to the application of this technique. Indeed, the dynamic programming equation can be written linearly in maxplus algebra. Therefore it is tempting to try to adapt the standard linear techniques (for example [9]) of linear system aggregation. This is possible and has been done in [15]. But some difficulties appear during this adaptation.

The maxplus analogs of linear space are an idempotent semimodule. As for modules, not all idempotent semimodules have a basis, possibly there does not exist a set of generators such that any vector of the semimodule has a unique set of coordinates. When this is the case, we say that the semimodule 
is free. Moreover, like in module theory, any surjective linear operator does not admit a linear inverse, when this is the case, the range space is called projective; dually when any injective linear application admits a linear inverse, we say that the domain is injective. To be able to build the analog of a linear projector on an image parallel to a kernel, we need a transversality condition that is the existence and uniqueness of the intersection of the kernel and the image (more precisely, the kernel introduced in the following defines a fibration and transversality means that each kernel fiber always intersects the image in a unique point). The transversality condition can be checked by means of linear algebra as soon as the ambient semimodule has some properties of projectiveness and injectiveness.

In a previous work [6], we have obtained necessary and sufficient conditions for the existence of a linear projector but the links with module theory were not given. In the present paper, we extend this result using assumptions of freeness on the ambient space and its order dual. This dual space is no longer defined as the set of continuous linear forms but it is rather derived from the definition of a scalar product using residuation. So doing, the bidual space is equal to the initial space. Apart from this improvement of the assumptions, the construction of the linear projector is the same and is recalled here.

There exist few works on these questions. We can find some results on semimodules in $[10,16]$. The application of linear projection to aggregation and coherency has been studied in [15]. We recall here some of these results, in particular, the notion of lumpability of dynamic programming equations.

\section{Ordered Algebraic Structures}

\subsection{Structure Definitions}

A semiring is a set $\mathcal{D}$ equipped with two operations $\oplus$ and $\otimes$ such that: $(\mathcal{D}, \oplus)$ is a commutative monoid whose zero is denoted by $\epsilon,(\mathcal{D}, \otimes)$ is a monoid whose unit is denoted by $e, \otimes$ is distributive with respect to $\oplus$, the zero is absorbing, that is $\epsilon \otimes a=a \otimes \epsilon=\epsilon$. A dioid is an idempotent semiring: $a \oplus a=a$. A semifield is a semiring in which nonzero elements have an inverse.

Example 1. The maxplus semifield $\mathbb{R}_{\max }$ is the set $\mathbb{R} \cup\{-\infty\}$ equipped with $\oplus=\max$ and $\otimes=+$. It is an idempotent semifield. .

A semimodule is the analogue of a module but defined on a semiring instead of a ring.

Example 2. $\mathbb{R}_{\max }^{n}$ is an idempotent semimodule.

Example 3. The set of $n \times n$ matrices with entries in $\mathbb{R}_{\max }$ is a dioid for the operations:

$$
(A \otimes B)_{i k}=\max _{j}\left\{A_{i j}+B_{j k}\right\}, \quad(A \oplus B)_{i j}=\max \left\{A_{i j}, B_{i j}\right\} .
$$


In a dioid, the operation $\oplus$ induces an order relation: $a \geq b \Longleftrightarrow a=$ $a \oplus b, \forall a, b \in \mathcal{D}$. Then $a \vee b=a \oplus b$. We say that a dioid $\mathcal{D}$ is complete if any arbitrary subset $B \subset \mathcal{D}$ has a supremum and if the product distributes with respect to suprema. When the supremum of a set $B$ belongs to $B$ we denote it by $\top B$.

Example 4. $\mathbb{R}_{\max }$ is not complete but $\overline{\mathbb{R}}_{\max }=\mathbb{R}_{\max } \cup\{+\infty\}$ is complete.

A complete dioid is a complete lattice. Indeed we can define the infimum of a set $A \subset \mathcal{D}$ by $\top\{d \in \mathcal{D} \mid d \leq a, \forall a \in A\}$. When the infimum of $A$ belongs to $A$, we denote it by $\perp A$.

\subsection{Residuated Mappings}

If $\mathcal{D}$ and $\mathcal{C}$ are complete lattices, we say that a map $f: \mathcal{D} \rightarrow \mathcal{C}$ is isotone if $a \geqslant b$ implies $f(a) \geqslant f(b)$. We say that $f$ is lower semicontinuous (lsc) if $f$ commutes with arbitrary suprema. We say that $f$ is residuated if the maximal element $f^{\sharp}(y) \triangleq \top\{x \in \mathcal{D}: f(x) \leqslant y\}$ exists for all $y \in \mathcal{C}$. The function $f^{\sharp}$ is called the residual of $f$. We say that a map $g: \mathcal{C} \mapsto \mathcal{D}$ is dually residuated if $g^{b}(x) \triangleq \perp\{y \in \mathcal{C}: g(y) \geqslant x\}$ exists for all $x \in \mathcal{C}$.

It is shown in [1] that $f$ is residuated iff $f$ is lsc with $f(\epsilon)=\epsilon$. Moreover, $f$ is residuated iff $f$ is isotone and there exists an isotone map $g: \mathcal{C} \rightarrow \mathcal{D}$ such that: $f \circ g \leqslant I_{\mathcal{C}}$ and $g \circ f \geqslant I_{\mathcal{D}}$.

The residual has the following properties: $f \circ f^{\sharp} \circ f=f ; f^{\sharp} \circ f \circ f^{\sharp}=f^{\sharp}$; $f$ is injective iff $f^{\sharp} \circ f=I_{\mathcal{D}}$ iff $f^{\sharp}$ is surjective; $f$ is surjective iff $f \circ f^{\sharp}=I_{\mathcal{C}}$ iff $f^{\sharp}$ is injective; $(h \circ f)^{\sharp}=f^{\sharp} \circ h^{\sharp} ; f \leqslant g$ iff $g^{\sharp} \leqslant f^{\sharp} ;(f \oplus g)^{\sharp}=f^{\sharp} \wedge g^{\sharp}$; $\left(f^{\sharp} \wedge g^{\sharp}\right)^{b}=f \oplus g$.

Example 5. The function $f: x \in \overline{\mathbb{R}}_{\max }^{2} \mapsto x_{1} \wedge x_{2} \in \overline{\mathbb{R}}_{\max }$ is isotone but not residuated. The function $f: x \in \overline{\mathbb{R}}_{\text {max }}^{2} \mapsto x_{1}^{2} \oplus x_{2}^{2} \triangleq \max \left(2 x_{1}, 2 x_{2}\right) \in \overline{\mathbb{R}}_{\max }$ is isotone, additive but nonlinear and residuated.
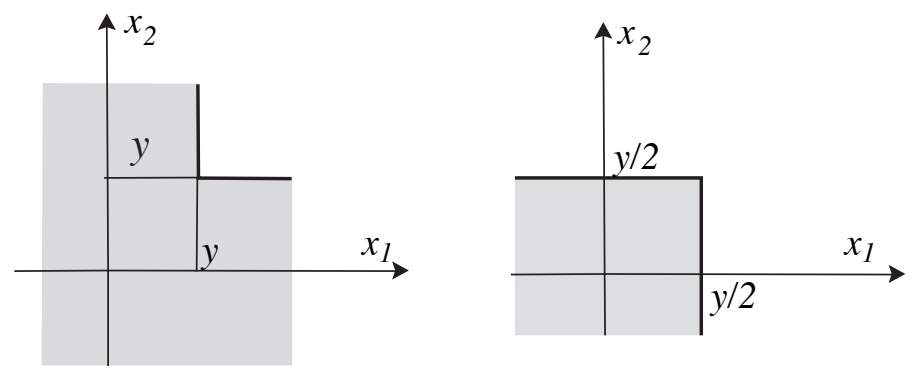

Fig. 1. $x_{1} \wedge x_{2} \leq y$ and $x_{1}^{2} \oplus x_{2}^{2} \leq y$ 
Example 6. If $A$ is a $m \times n$ matrix with entries in $\overline{\mathbb{R}}_{\max }$, the morphism $x \in$ $\overline{\mathbb{R}}_{\text {max }}^{m} \mapsto A x \in \overline{\mathbb{R}}_{\text {max }}^{n}$ is residuated: the maximal element of the set $\left\{x \in \overline{\mathbb{R}}_{\text {max }}^{n} \mid\right.$ $A x \leqslant b\}$ exists, it is denoted $A \backslash b$ with $(A \backslash b)_{j}=\bigwedge_{i} A_{i j} \backslash b_{i}$. Indeed:

$$
\begin{aligned}
A x \leqslant b & \Leftrightarrow \bigoplus_{j} A_{i j} x_{j} \leqslant b_{i}, \forall j \Leftrightarrow A_{i j} x_{j} \leqslant b_{i}, \forall i, j \\
& \Leftrightarrow x_{j} \leqslant A_{i j} \backslash b_{i}, \forall i, j \Leftrightarrow x_{j} \leqslant \bigwedge_{i} A_{i j} \backslash b_{i}, \forall j .
\end{aligned}
$$

Thus $A \backslash b=\left(-A^{t}\right) \odot b$ where $\odot$ denotes the minplus matrix product. In the same way, we can compute $A \backslash B, B / A, C \backslash A / B$ for matrices with compatible dimensions.

For example $A \backslash B$ is the largest matrix such that $A X \leq B$ and, if there exists $X$ such that $A X=B$, we have $A(A \backslash B)=B$. Moreover $B \mapsto A \backslash B$ is an $\wedge$ morphism.

\section{Idempotent Semimodules}

In this section we extend the notion of projective module and injective module to projective and injective idempotent semimodule.

\subsection{Projective Idempotent Semimodules}

Definition 1. A complete idempotent semimodule $\mathcal{P}$ is projective if for all complete idempotent semimodules $\mathcal{U}$ and $\mathcal{X}$ and for all morphisms $A: \mathcal{P} \rightarrow \mathcal{X}$ and $B: \mathcal{U} \rightarrow \mathcal{X}$ such that $\operatorname{im}(A) \subset \operatorname{im}(B)$ there exists a morphism $R: \mathcal{P} \rightarrow \mathcal{U}$ such that $A=B R$.

This is of course the analog of the classical notion in module theory. The following proposition shows that free complete idempotent semimodules are projective and the factor $R$ can be computed by residuation.

Proposition 1. Given the free idempotent complete semimodule $\mathcal{P}$, the two idempotent complete semimodules $\mathcal{U}, \mathcal{X}$, the morphism $B: \mathcal{U} \rightarrow \mathcal{X}$ and the morphism $A: \mathcal{P} \rightarrow \mathcal{X}$ such that $\operatorname{im}(A) \subset \operatorname{im}(B)$, we have:

$$
A=B(B \backslash A) .
$$

The existence of a morphism $R$ such that $A=B R$ follows from the classical argument [1, Th. 8.6] The maximal $R$ is equal to $(B \backslash A)$. In the case of matrices $(B \backslash A)$ has been computed as in Example 6 .

Example 7. $\mathcal{X}=\operatorname{im}(B)$ subsemimodule of $\overline{\mathbb{R}}_{\max }^{2}$ with $B=\left[\begin{array}{ll}1 & e \\ e & 1\end{array}\right]$ is not free. 
The vector $\left[\begin{array}{l}1 \\ e\end{array}\right]$ can also be written $\left[\begin{array}{l}1 \\ e\end{array}\right] \oplus(-1)\left[\begin{array}{l}e \\ 1\end{array}\right]$ and therefore has not a unique set of coordinates on the generating family $\left\{\left[\begin{array}{l}1 \\ e\end{array}\right],\left[\begin{array}{l}e \\ 1\end{array}\right]\right\}$. However, $\operatorname{im}(B)$ is projective because $B$ has a generalized inverse $B^{g}$, meaning that $B B^{g} B=B$. We can take: $B^{g}=\left[\begin{array}{ll}-1 & -2 \\ -2-1\end{array}\right]$.

\subsection{Injective Idempotent Semimodules}

To discuss injective idempotent semimodules, it is necessary to extend the notion of morphism kernel. Indeed, since in a semiring the general linear equation $A x=A^{\prime} x$ cannot be reduced to $A^{\prime \prime} x=\epsilon$, the standard kernel definition is not very useful. Instead, we introduce the fibration of the domain by the injectivity classes of a morphism.

Definition 2. Given two semimodules $\mathcal{X}$ and $\mathcal{Y}$ and a morphism $C: \mathcal{X} \rightarrow \mathcal{Y}$, we define the equivalence relation $\operatorname{ker}(C)$ on $\mathcal{X}$ by $x \sim x^{\prime}$ modulo $\operatorname{ker}(C)$ if $C x=C x^{\prime}$.

We say that $\operatorname{ker}(C) \subset \operatorname{ker}(A)$ when the fibration induced by $\operatorname{ker}(C)$ is a subfibration of that of $\operatorname{ker}(A)$, that is, when $C x=C x^{\prime}$ implies $A x=A x^{\prime}$. With this kernel definition, we can extend the notion of injective modules to injective semimodules.

Definition 3. A complete idempotent semimodule $\mathcal{I}$ is injective if for all complete idempotent semimodules $\mathcal{Y}$ and $\mathcal{X}$ and for all morphisms $A: \mathcal{X} \rightarrow \mathcal{I}$ and $C: \mathcal{X} \rightarrow \mathcal{Y}$ satisfying $\operatorname{ker}(C) \subset \operatorname{ker}(A)$ there exists a morphism $L: \mathcal{Y} \rightarrow \mathcal{I}$ such that $A=L C$.

To understand the duality between projective and injective idempotent semimodules, it is useful to introduce a duality on idempotent semimodules based on residuation.

If $\mathcal{X}$ is a complete idempotent semimodule on the idempotent semiring $\mathcal{D}$, we call (order) dual of $\mathcal{X}$, the semimodule on the semiring $\mathcal{D}$, denoted $\mathcal{X}^{\sharp}$, with underlying set $\mathcal{X}$, addition $\left(x \in \mathcal{X}, x^{\prime} \in \mathcal{X}\right) \mapsto\left(x \wedge x^{\prime}\right) \in \mathcal{X}$ (where $\wedge$ is relative to the natural order on $\mathcal{X}$ induced by $\oplus)$ and action $(\lambda \in \mathcal{D}, x \in \mathcal{X}) \mapsto$ $x / \lambda \in \mathcal{X}$. The semimodule property of $\mathcal{X}^{\sharp}$ comes easily from the residuation properties.

Proposition 2 ([7] Prop. 4). For all complete idempotent semimodules $\mathcal{X}$, $\left(\mathcal{X}^{\sharp}\right)^{\sharp}=\mathcal{X}$.

Associated with a semimodule morphism $C: \mathcal{X} \rightarrow \mathcal{Y}$, the residuation operation defines the semimodule morphism $C^{\sharp}: \mathcal{Y}^{\sharp} \rightarrow \mathcal{X}^{\sharp}$ with which we can characterize the kernel inclusions. 
Proposition 3. Given two idempotent complete semimodules $\mathcal{X}, \mathcal{Y}$, two morphisms $A$ and $C$ from $\mathcal{X}$ to $\mathcal{Y}$, we have:

$$
\operatorname{ker}(C) \subset \operatorname{ker}(A) \Leftrightarrow \operatorname{im}\left(A^{\sharp}\right) \subset \operatorname{im}\left(C^{\sharp}\right) .
$$

Proof. If $\operatorname{im}\left(A^{\sharp}\right) \subset \operatorname{im}\left(C^{\sharp}\right)$, any point in $\operatorname{im}\left(A^{\sharp}\right)$ does not move when projected on $\operatorname{im}\left(C^{\sharp}\right)$, which translates into the equality $C^{\sharp} C A^{\sharp}=A^{\sharp}$. By pre- and postcomposition with $A$ of both sides of this equality, one gets that

$$
A C^{\sharp} C A^{\sharp} A=A A^{\sharp} A=A .
$$

We have $A C^{\sharp} C A^{\sharp} A \geq A C^{\sharp} C \geq A$ because both $A^{\sharp} A$ and $C^{\sharp} C$ are greater than the identity. Finally, equality holds throughout and we have proved that $A=A C^{\sharp} C$, from which it is clear that $\operatorname{ker}(C) \subset \operatorname{ker}(A)$.

Conversely, it is easily checked that $C^{\sharp} C x$ is equivalent to $x$ modulo $\operatorname{ker}(C)$. Moving in the same class modulo $\operatorname{ker}(C)$ implies moving in the same class modulo $\operatorname{ker}(A)$ from the assumption. Therefore $A=A C^{\sharp} C$. Then, pre and post-composition with $A^{\sharp}$ shows that

$$
A^{\sharp} A C^{\sharp} C A^{\sharp}=A^{\sharp} A A^{\sharp}=A^{\sharp}
$$

and $A^{\sharp} A C^{\sharp} C A^{\sharp} \geq C^{\sharp} C A^{\sharp} \geq A^{\sharp}$ which shows that equality holds throughout. Therefore $A^{\sharp}=C^{\sharp} C A^{\sharp}$ which shows that $\operatorname{im}\left(A^{\sharp}\right) \subset \operatorname{im}\left(C^{\sharp}\right)$.

Theorem 1. An idempotent complete semimodule is injective if and only if its (order) dual is projective.

Proof. If $\mathcal{I}^{\sharp}$ is projective and the morphisms $A: \mathcal{X} \rightarrow \mathcal{I}$ and $C: \mathcal{X} \rightarrow \mathcal{Y}$ satisfy $\operatorname{ker}(C) \subset \operatorname{ker}(A)$ then $\operatorname{im}\left(A^{\sharp}\right) \subset \operatorname{im}\left(C^{\sharp}\right)$ and because $\mathcal{I}^{\sharp}$ is projective there exists a morphism $L^{\sharp}: \mathcal{I}^{\sharp} \rightarrow \mathcal{X}^{\sharp}$ such that $A^{\sharp}=C^{\sharp} L^{\sharp}$ which implies $A=L C$ thanks to the residuation properties.

Conversely, if $\mathcal{I}$ is injective, consider the morphisms $A^{\sharp}: \mathcal{I}^{\sharp} \rightarrow \mathcal{X}^{\sharp}$ and $C^{\sharp}: \mathcal{X}^{\sharp} \rightarrow \mathcal{Y}^{\sharp}$ such that $\operatorname{im}\left(A^{\sharp}\right) \subset \operatorname{im}\left(C^{\sharp}\right)$ which implies $\operatorname{ker}(C) \subset \operatorname{ker}(A)$ which implies $A=L C$ since $\mathcal{I}$ is injective and therefore $A^{\sharp}=C^{\sharp} L^{\sharp}$ which shows that $\mathcal{I}^{\sharp}$ is projective.

Proposition 4. Given a complete idempotent semimodule $\mathcal{I}$ with a free dual, two complete idempotent semimodules $\mathcal{Y}, \mathcal{X}$ and two morphisms $A: \mathcal{X} \rightarrow \mathcal{I}$, $C: \mathcal{X} \rightarrow \mathcal{Y}$ with $\operatorname{ker}(C) \subset \operatorname{ker}(A)$ we have:

$$
A=(A / C) C .
$$

It is useful to remark that the dual of a free semimodule is not always free. Example 8. $\overline{\mathbb{R}}_{\text {max }}^{n}$ is free and its dual $\overline{\mathbb{R}}_{\text {min }}^{n}$ is free and therefore these two semimodules are projective and injective.

Example 9. $\overline{\mathbb{R}}_{\max }^{+}=\left(\mathbb{R}^{+} \cup\{-\infty,+\infty\}\right.$, max, +$)$ is a complete idempotent semimodule on $\overline{\mathbb{R}}_{\text {max }}^{+}$which is free, its basis is $\{0\}$, but its dual is not free. 


\section{Projectors}

In the following, the sets $\mathcal{U}, \mathcal{X}$ and $\mathcal{Y}$ will be complete idempotent semimodules. Moreover, we will suppose that $\mathcal{X}$ and its dual are free so that we can test the image or kernel inclusions by (1) or (2). Based on those assumptions, following [6], we build linear projectors on subsemimodules of $\mathcal{X}$ that can be described as images of regular morphisms.

We say that $B$ is regular if there exists a generalized inverse $B^{g}$ satisfying: $B=B B^{g} B$. Then there exists a largest generalized inverse equal to $B \backslash B / B$. Therefore we have:

$$
B=B(B \backslash B / B) B
$$

if and only if $B$ is regular. Indeed, the existence of $B^{g}$ implies the existence of $X$ such that $B=B X B$ and therefore the largest $X$ such that $B \geq B X B$ (which is equal to $B \backslash B / B$ ) satisfies the equality.

Moreover, with every generalized inverse, we can associate a generalized reflexive inverse. Indeed, $B^{r} \triangleq B B^{g} B$ is an inverse that satisfies

$$
B=B B^{r} B, \quad B^{r}=B^{r} B B^{r},
$$

which are the relations which define the reflexive inverses.

\subsection{Projector on $\operatorname{Im}(B)$}

Proposition 5. There exists a linear projector $Q$ on $\operatorname{im}(B)$ iff $B$ is regular.

By linear projector we mean a projector that is a morphism of complete idempotent semimodules.

Proof. If $Q$ is a linear projector on $\operatorname{im}(B)$, we have $Q B=B$. Since $B$ and $Q$ have the same image, by (1) we have $Q=B(B \backslash Q)$, which implies $B=$ $B(B \backslash Q) B$. Therefore we can take $B^{g}=B \backslash Q$ as generalized inverse. Thus $B$ is regular.

Conversely if $B^{g}$ is a generalized inverse of $B$ then $Q=B B^{g}$ is a linear projector on $\operatorname{im}(B)$.

In order to prepare the transition with the next section, it is useful to give other forms to the projector $Q$

Proposition 6. If $B$ is regular and $B^{r}$ is a generalized reflexive inverse of $B$, we have

$$
Q=B B^{r}=\left(B /\left(B^{r} B\right)\right) B^{r}=B\left(\left(B^{r} B\right) \backslash B^{r}\right) .
$$

Proof. From $B=B B^{r} B$, we deduce from the residuation properties that $B=\left(B /\left(B^{r} B\right)\right) B^{r} B$. Similarly from $B^{r}=B^{r} B B^{r}$, we deduce $B^{r}=$ $B^{r} B\left(\left(B^{r} B\right) \backslash B^{r}\right)$. Let $P$ defined by $P \triangleq\left(B /\left(B^{r} B\right)\right) B^{r}$ we have $P=$ $\left(B /\left(B^{r} B\right)\right) B^{r} B\left(\left(B^{r} B\right) \backslash B^{r}\right)=B\left(\left(B^{r} B\right) \backslash B^{r}\right)$. 
Moreover $P B=B, B^{r} P=B^{r}$ and $P^{2}=\left(B /\left(B^{r} B\right)\right) B^{r} B\left(\left(B^{r} B\right) \backslash B^{r}\right)=$ $\left(B /\left(B^{r} B\right)\right) B^{r}=P$, therefore $P$ is a projector on $\operatorname{im}(B)$ and since $B^{r} P=B^{r}$ the projection is parallel to $\operatorname{ker}\left(B^{r}\right)$. $P$.

Finally: $Q=B B^{r}=\left(B /\left(B^{r} B\right)\right) B^{r} B B^{r} B\left(\left(B^{r} B\right) \backslash B^{r}\right)=\left(B /\left(B^{r} B\right)\right) B^{r}=$

\subsection{Projector on $\operatorname{Im}(B)$ parallel to $\operatorname{Ker}(C)$}

To define a projector on $\operatorname{im}(B)$ parallel to $\operatorname{ker}(C)$, we need a transversality condition, that is, each equivalent class of $\operatorname{ker}(C)$ intersects $\operatorname{im}(B)$ in exactly one point (for all $x$ there exists a unique $x^{\prime}$ such that $C x=C x^{\prime}$ with $x^{\prime}: x^{\prime}=$ $B u)$. The following theorem gives a test for transversality.

Theorem 2 ([6] Th.8 and 9). The three following assertions are equivalent:

1. $\operatorname{ker}(C B)=\operatorname{ker}(B)$ and $\operatorname{im}(C B)=\operatorname{im}(C)$, that is:

$$
B=(B /(C B)) C B, \quad C=C B((C B) \backslash C) .
$$

2. There exists a linear projector $P$ on $\operatorname{im}(B)$ parallel to $\operatorname{ker}(C)$ :

$$
P B=B, C P=C, P^{2}=P=(B /(C B)) C=B((C B) \backslash C) .
$$

3. $\operatorname{im}(B)$ is transverse to $\operatorname{ker}(C)$.

Let us only show that the first proposition implies the third one.

The existence of the intersection follows from $C=C B((C B) \backslash C)$, indeed $C x=C B((C B) \backslash C) x$ therefore $y=B((C B) \backslash C) x$ belongs to $\operatorname{im}(B)$ and is in the same $\operatorname{ker}(C)$-class as $x$.

The uniqueness of the intersection follows from $B=(B /(C B)) C B$, indeed: $C B u=C B u^{\prime}$ implies $(B /(C B)) C B u=(B /(C B)) C B u^{\prime}=B u=B u^{\prime}$.

Example 10. With $\mathcal{U}=\mathcal{X}=\mathcal{Y}=\mathbb{R}_{\max }^{2}$ and

$$
B=C=\left[\begin{array}{cc}
e & -1 \\
-1 & e
\end{array}\right],
$$

we have $B=B / B$ and it follows that $P=B$. More generally, for any $E$, as soon as $B=E / E$ we have: $B=B / B=B^{2}=B \backslash B=B \backslash B / B=B^{r}=P$.

Example 11. For $\mathcal{X}=\mathbb{R}_{\max }^{2}, \mathcal{U}=\mathcal{Y}=\mathbb{R}_{\max }$ and $B$ and $C$ given by:

$$
B=\left[\begin{array}{l}
e \\
e
\end{array}\right], \quad C=[e-1] .
$$

the projector is given in Figure 3 . 


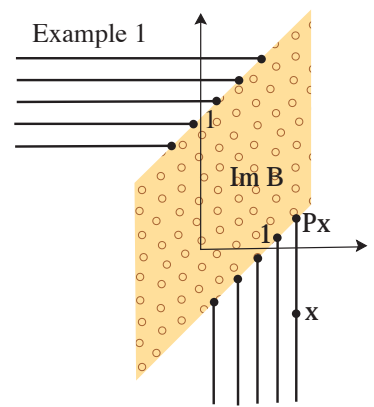

Fig. 2. Projection on $\operatorname{im}(B)$ parallel to $\operatorname{ker}(B)$.

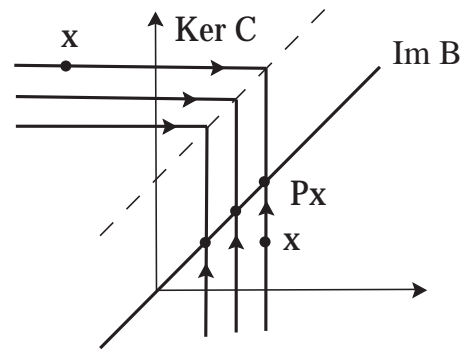

Fig. 3. Projection on $\operatorname{im}(B)$ parallel to $\operatorname{ker}(C)$.

Example 12. For the following $B$

$$
B=\left[\begin{array}{lll}
a & e & e \\
e & a & e \\
e & e & a
\end{array}\right]
$$

We have $B \backslash B$

$$
B \backslash B=\left[\begin{array}{ccc}
e & -|a| & -|a| \\
-|a| & e & -|a| \\
-|a| & -|a| & e
\end{array}\right] .
$$

With some calculation one can show that:

$$
B^{\sharp} B\left[\begin{array}{l}
x \\
y \\
z
\end{array}\right]=(B \backslash B)\left[\begin{array}{l}
x \\
y \\
z
\end{array}\right] \oplus \chi\left[\begin{array}{l}
y \wedge z \\
x \wedge z \\
x \wedge y
\end{array}\right],
$$

where $\chi=e$ if $a<e$ and $\chi=\epsilon$ otherwise.

Then we see that $B^{\sharp} B=(B \backslash B)$ when $a \geq e$. We can verify that the case $a \geq e$ is precisely the case where the matrices $B$ are regular.

Example 13. The projector shown in Figure 4 is defined with the following $B$ and $C$ : 


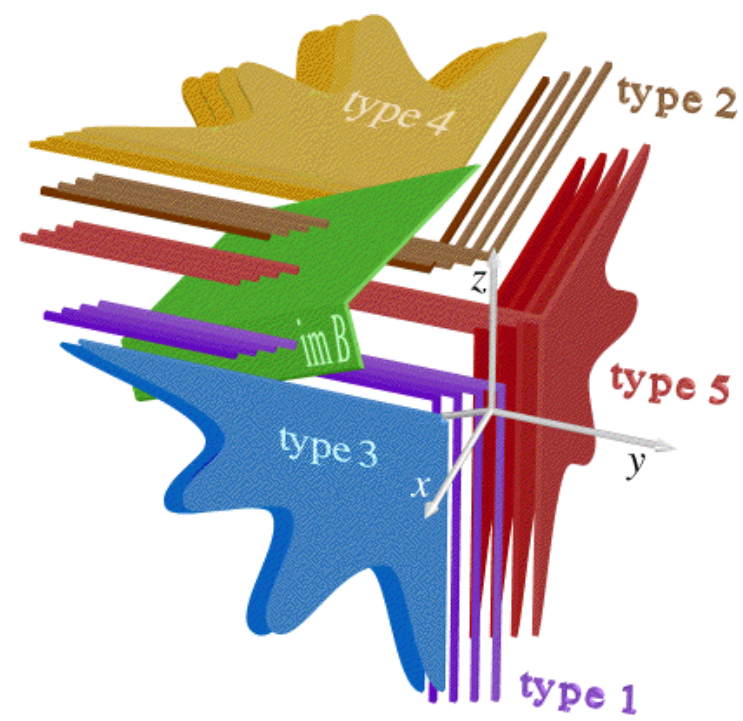

Fig. 4. Projection on $\operatorname{im}(B)$ parallel to $\operatorname{ker}(C)$ in dimension 3.

$$
B=\left[\begin{array}{cc}
0 & 1 \\
0.5 & 0 \\
2 & 1
\end{array}\right], \quad C=\left[\begin{array}{lll}
0 & 0 & 0 \\
2 & 1 & 0
\end{array}\right]
$$

We see on this example that the fiber shapes can be different. In this example, we have five kinds of fiber having three different shapes.

\section{Aggregation and Coherency}

Thanks to the projectors defined in the previous section, the results about aggregation and coherency given in [9] can be extended to the case of maxplus algebra. This means that the aggregation tools used in the theory of linear systems can be applied to aggregation of dynamic programming problems or Hamilton-Jacobi equations. In this section, we recall some results given in [15].

Given $\mathcal{X}$, a complete free idempotent semimodule with free dual, we consider the endomorphism $A: \mathcal{X} \rightarrow \mathcal{X}$ and the dynamic system $X_{n+1}=A X_{n}$. We say that $A$ is aggregable by $C: \mathcal{X} \rightarrow \mathcal{Y}$ (regular morphism on the idempotent complete semimodule $\mathcal{Y}$ ) if there exists $A_{C}$ such that $C A=A_{C} C$. Then, $Y_{n} \triangleq C X_{n}$ satisfies the aggregate dynamics $Y_{n+1}=A_{C} Y_{n}$.

Proposition 7. If $C$ is regular, $A$ is aggregable by $C$ iff there exists $B$ such that the projector on $\operatorname{im}(B)$ parallel to $\operatorname{ker}(C)$ satisfies $P A=P A P$.

Proof. Since $C$ is regular, there exists $B$ and $P$ with $P=B((C B) \backslash C)=$ $(B /(C B)) C$. 
- Sufficiency $\left(P A=P A P \Rightarrow C A=A_{C} C\right)$ :

$C A=C P A=C P A P=C A P=C A(B /((C B))) C=[C A(B /(C B))] C$,

and we have $A_{C}=C A(B /(C B))$.

- Necessity $\left(P A=P A P \Leftarrow C A=A_{C} C\right)$ :

$$
\begin{aligned}
P A & =(B /(C B)) C A=(B /(C B)) A_{C} C=(B /(C B)) A_{C} C P \\
& =(B /(C B)) C A P=P A P .
\end{aligned}
$$

In a similar way, we say that $B$, assumed regular, is coherent with $A$ if there exists $A_{B}$ such that: $A B=B A_{B}$. In this case, if $X_{0}=B U_{0}$, then $X_{n}=B U_{n}$ is defined by the aggregate dynamics $U_{n+1}=A_{B} U_{n}$.

Proposition 8. If $B$ is regular, $B$ is coherent with $A$ iff there exists $C$ such that the $P$ projector on $\operatorname{im}(B)$ parallel to $\operatorname{ker}(C)$ satisfies: $A P=P A P$.

To show an analogy with the lumpability of Markov chains [12], we can specialize the previous results to the case when $C$ is defined as the characteristic function of a partition.

Let us suppose that $\mathcal{X}=\overline{\mathbb{R}}_{\min }^{n}$. Consider a partition $\mathcal{U}=\left\{J_{1}, \ldots, J_{p}\right\}$ of $F=\{1, \cdots, n\}$ and its characteristic matrix:

$$
U_{i J}=\left\{\begin{array}{ll}
e & \text { if } i \in J, \\
\epsilon & \text { si } i \notin J,
\end{array} \forall i \in F, \forall J \in \mathcal{U} .\right.
$$

If $w \in \overline{\mathbb{R}}_{\text {min }}^{n}$ is a cost (which is analogous to a probability, that is if $w$ satisfies $g w=e$ with $g$ a row vector with all entries equal to $e$ ) the conditional cost with respect to $\mathcal{U}$ is defined by:

$$
\left(w^{\mathcal{U}}\right)_{i J}=\frac{w_{j}}{\bigoplus_{j \in J} w_{j}}, \quad \forall j, J
$$

Clearly we have:

$$
w^{\mathcal{U}}=W U S^{-1}, \text { with } S \triangleq U^{t} W U, W=\operatorname{diag}(w) .
$$

If $A$ is the transition cost of a Bellman chain (that is $g A=g$ ), we say that $A$ is $\mathcal{U}$-lumpable if $A$ is aggregable with $C=U^{t}$.

If $A$ admits a unique invariant cost $A w=w$, and if $A$ is $\mathcal{U}$-lumpable, we can take $P=B C$ with $B=w^{\mathcal{U}}$ for projection on $\operatorname{im}(B)$ parallel to $\operatorname{ker}(C)$ since $C B=U^{t} W U S^{-1}=S S^{-1}=I$ (the identity matrix $I$ is the matrix with a diagonal of $e$ and $\epsilon$ elsewhere).

In this case, looking at the meaning of $C A=A_{C} C$, we have:

Proposition 9 ([15] Th.20). A is lumpable iff:

$$
\bigoplus_{k \in K} a_{k j}=\bar{a}_{K J}, \forall j \in J, \forall J, K \in \mathcal{U}
$$




\section{References}

1. T.S. BLYTH (1990), Module theory. Oxford Science Publications.

2. T.S. BLYTH, M.J JANOWITZ (1972), Residuation theory. Pergamon Press, Oxford.

3. Z.Q. CAO, K.H. KIM, F.W. ROUSH (1984), Incline Algebra and Applications, Hellis Horwood.

4. F. BACCELLI, G. COHEN, G.J. OLSDER, and J.-P. QUADRAT (1992), Synchronization and Linearity, Wiley and http://www-rocq.inria.fr/metalau/cohen/SED/book-online.html .

5. G. COHEN, S. GAUBERT, J.P. QUADRAT (1996), Kernels, images and projections in dioids. Proceedings of WODES'96, Edinburgh and http://www-rocq.inria.fr/metalau/quadrat/kernel.pdf .

6. G. COHEN, S. GAUBERT, J.P. QUADRAT (1997). Linear Projectors in the max-plus algebra, 5th IEEE-Mediterranean Conf. Paphos, Cyprus and http://www-rocq.inria.fr/metalau/quadrat/projector.pdf .

7. G. COHEN, S. GAUBERT, J.P. QUADRAT (2004), Duality and Separation Theorems in idempotent Semimodules, Linear Algebra and its Applications N. 379 pp. 395-422 and arXiv:math.FA/0212294.

8. R.A. CUNNINGHAME-GREEN (1979), Minimax Algebras. L.N. in Economics and Math. Systems, Springer Verlag.

9. F. DELEBECQUE, P. KOKOTOVIC, J.-P. QUADRAT (1984). Aggregation and coherency in networks and Markov chains. Int. Jour. of Control N. 35 and http://www-rocq.inria.fr/metalau/quadrat/Aggregation.pdf .

10. J.S. GOLAN (1992), The theory of semirings with applications in mathematics and theretical computer science, V.54, Longman Sci \& Tech.

11. K.H. KIM (1982), Boolean Matrix Theory and Applications, Macell Dekker, New York.

12. J.G. KEMENY, J.L. SNELL, A.W. KNAP (1976), Finite Markov chains, Springer-Verlag.

13. F.P. KELLY (1976). Reversibility and stochastic networks. Wiley, (1979).

14. V. MASLOV, S. SAMBORSKII (1992), editors, Idempotent Analysis V.13 of Adv. in Sov. Math. AMS, RI.

15. J.-P. QUADRAT and MAX-PLUS (1997), Min-plus linearity and statistical mechanics Markov Processes and Related Fields, N.3, http://www-rocq.inria.fr/metalau/quadrat/MecaStat.pdf

16. E. WAGNEUR (1991) Moduloids and Pseudomodules, Dimension Theory, Discrete Math. V.98, pp. 57-73.

Other related papers and informations about maxplus algebra are available from the web site: http://maxplus.org . 
